\title{
An Unusual Timeline of Nivolumab-Induced Vitiligo in a Patient with Melanoma
}

\author{
Renat Ahatov, BSA ${ }^{1}$, Allison Good, $\mathrm{MD}^{2}$, Lindy Ross, $\mathrm{MD}^{2}$ \\ ${ }^{1}$ The University of Texas Medical Branch School of Medicine in Galveston, TX \\ ${ }^{2}$ The University of Texas Medical Branch Department of Dermatology in Galveston, TX.
}

\section{ABSTRACT}

Vitiligo, manifested by skin hypopigmentation, is an autoimmune disorder of the skin due to the autoimmune destruction of melanocytes. Nivolumab, which is a programmed cell death-1 receptor inhibitor, is a well-known therapy for melanoma. Nivolumab-induced vitiligo has been described in literature, explained by destruction of non-cancerous melanocytes. This is considered a favorable response, due to a correlated stronger immune response against tumor cells. The average onset of the vitiligo is reported to be 5.2 months after starting the therapy, with a maximum reported onset being 9 months. We present a 52 -year-old male patient whose initial vitiligo presentation occurred a year after starting the therapy and has continued for months after concluding Nivolumab therapy.

\section{INTRODUCTION}

Vitiligo is an autoimmune disorder of the skin, marked by depigmentation of the skin, due to the autoimmune loss of melanocytes. Vitiligo has been reported to occur in up to $25 \%$ of the patients on programmed cell death-1 receptor (PD1) inhibitors. ${ }^{1}$ PD-1 ligand is produced by some cancer cells as a means of immune system evasion. PD-1 ligand binds to PD-1, which leads to T-cell inactivation that allows the cancer to proliferate unchecked by the immune system. Nivolumab is a humanized IgG4 anti-PD-1 monoclonal antibody that prevents T-cell inactivation. It is an FDAapproved treatment for unresectable/metastatic melanoma, along with various other malignancies. ${ }^{2}$ The vitiligo seen with Nivolumab has been theorized to be due to the destruction of non-cancerous melanocytes by activated T-cells. This side effect of Nivolumab has been associated with more favorable outcomes in melanoma, as it correlates to a strong immune response against tumor cells. A previous study found that vitiligo typically presents 2 to 9 months after starting Nivolumab, with an average presentation onset of 5.2 months. ${ }^{3}$ We present a case of a patient who was started on Nivolumab for his melanoma and developed vitiligo one year after beginning PD-1 inhibitor therapy around the time of his final Nivolumab cycle, which is later onset than previously reported in the literature. In addition, the vitiligo continued to progress after treatment with nivolumab was completed, which has not been previously reported with nivolumabassociated vitiligo.

\section{CASE REPORT}

January 2022 Volume 6 Issue 1

(c) 2022 THE AUTHORS. Published by the National Society for Cutaneous Medicine. 


\section{SKIN}

A 52-year-old male with superficial spreading melanoma stage IIIA T2N2M0 was treated with wide local excision of the tumor and sentinel lymph node biopsy. Two lymph nodes were involved with metastatic melanoma, and Nivolumab therapy was initiated. The patient was started on Nivolumab therapy one month after the excision and completed the therapy one year later with no evidence of tumor recurrence or metastasis. Two months after completion of Nivolumab, the patient was seen at a follow-up visit. The patient noted the development of an asymptomatic depigmented patch on his right cheek that started one year after starting the Nivolumab therapy, about the time the therapy was completed (Figure 1).

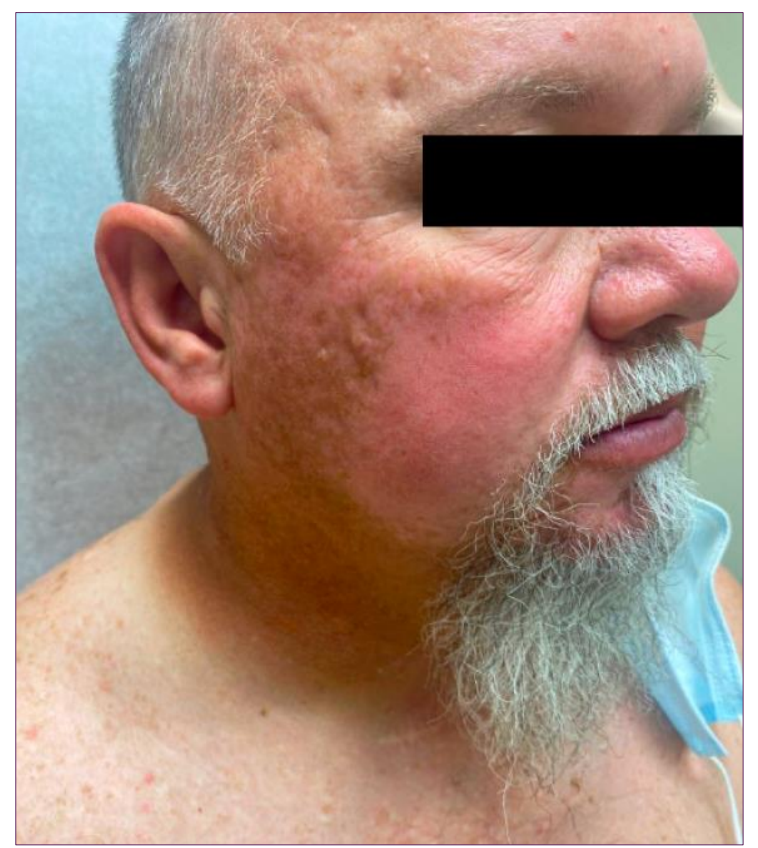

Figure 1. Depigmentation noted on the right cheek approximately 14 months after initiating Nivolumab

The patient's thyroid stimulating hormone level was within normal limits. He had no personal or family history of vitiligo, and he had no halo nevi. The patient was seen for follow-up three months later, at which point he noted that the depigmented patch had continued to spread to his forehead, scalp, and left cheek (Figure 2). He also reported that the right cheek became erythematous with sun exposure. On physical exam, depigmented patches were present on both cheeks and forehead, with erythema within the depigmented patch of the right cheek. Approximately four months later, the patient did not see significant improvement in the depigmentation and had a new depigmented macule on his left forearm. No sign of melanoma recurrence has been noted and no evidence of other immune-related side effects were noted. The patient is currently continuing to be closely monitored for presumed post-Nivolumab vitiligo. Patient declined treatment with topical steroids, but has gotten low levels of ambient sun exposure and has noticed re-pigmentation of the cheek and scalp at one-year follow-up appointment.

\section{DISCUSSION}

PD-1 inhibitors and their associations with various cutaneous phenomena have been well described in the literature. Some of these include lichenoid dermatosis, eczema, vitiligo, and Steven-Johnson syndrome. ${ }^{4}$ The pathophysiology of vitiligo is the 


\section{SKIN}

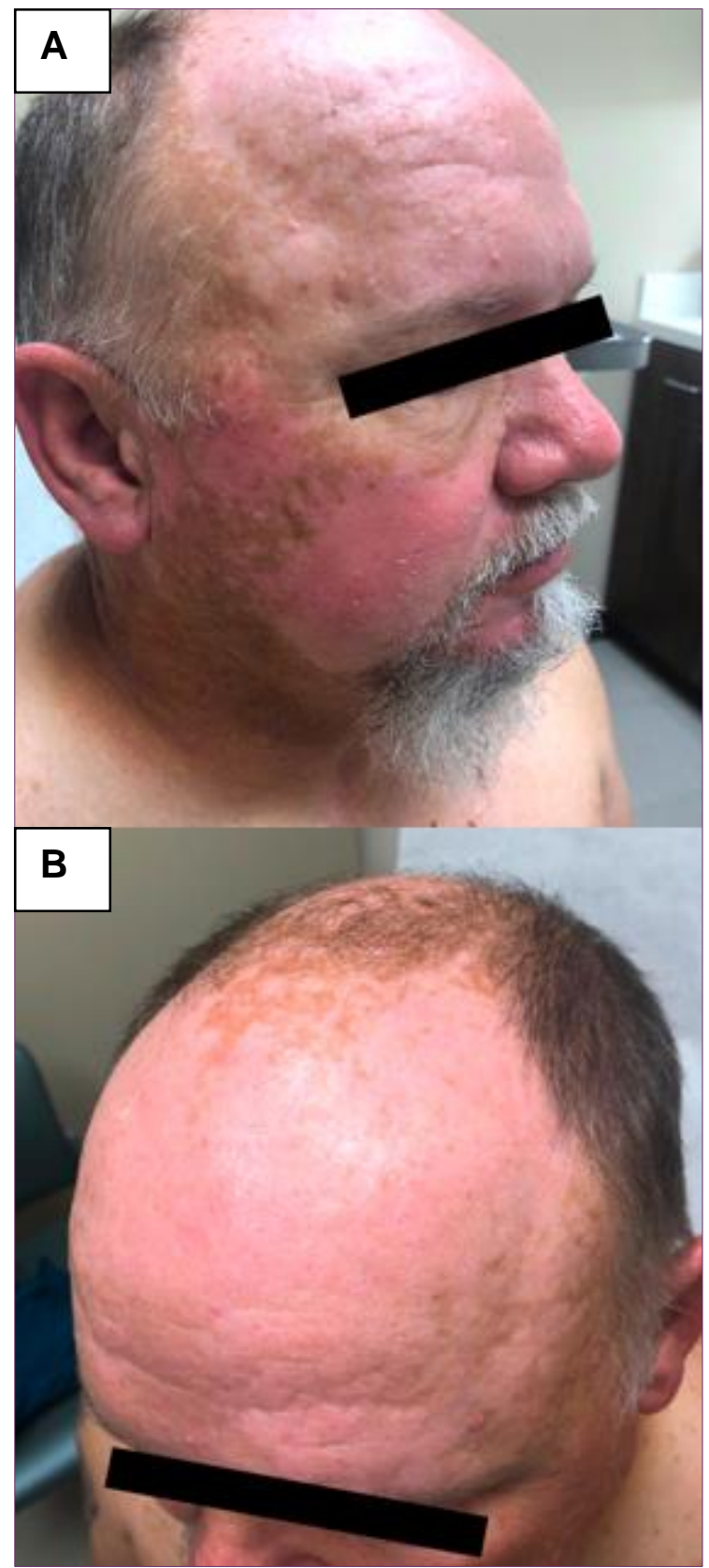

Figure 2. A) Increased depigmentation noted on the right cheek and newly developed depigmentation on B) forehead and scalp approximately 17 months after initiating the therapy.

autoimmune destruction of epidermal melanocytes caused by T-cell infiltration. Vitiligo is often associated with other autoimmune diseases such as lupus, autoimmune thyroiditis, and Addison disease, but it can also occur with immunotherapy usage. ${ }^{5,6}$ With PD-1 inhibitors, it is postulated that the healthy melanocytes are targeted due to the overlapping antigens on the normal melanocytes with those on the melanoma cells. Therefore, it is associated with a tumor response in melanoma therapy, as it indicates an adequate response to the PD-1 inhibitor therapy. ${ }^{3}$ Vitiligo is most commonly seen when Nivolumab is used for melanomas. However, cases of vitiligo in other cancers treated by Nivolumab have also been reported, including lung cancer, renal cancer, and leukemia. 4,5,7 The average time until vitiligo presentation after starting Nivolumab therapy was reported to be 5.2 months in a prior study. While the sample size in that study was nine patients, previous cases in the literature report vitiligo developing 9 months or less after the initiation of Nivolumab. ${ }^{3,5}$ In our patient, the vitiligo on his right cheek began about one year after starting Nivolumab and continued to progress after the completion of Nivolumab therapy. While it is possible that the vitiligo began less than one year after beginning therapy as this timeframe was patient-reported, the continued progression of the vitiligo while no longer on Nivolumab is unusual and may be explained by sustained antitumor $T$ cell activity induced by Nivolumab therapy. It is also possible that melanoma itself may cause vitiligolike lesions, but the patient continues to have no signs of disease progression with regular follow-up.

Currently, there are no guidelines for the treatment for this post-immunotherapy vitiligo, but observation and treatment with topical corticosteroids, calcineurin inhibitors, and phototherapy can be recommended. Although vitiligo is a 
benign condition, it can be psychologically stressful to the patient, especially as it relates to a serious disease such as melanoma. Therefore, psychiatric support, such as counseling, can also be offered as needed. ${ }^{8}$ In this case, the patient declined treatment because the vitiligo was not cosmetically bothersome to him. Close observation will be continued.

\section{CONCLUSION}

This case highlights the possibility of post-immunotherapy vitiligo occurring much later than the previously reported average of 5.2 months after starting Nivolumab therapy. The case is also unique in its continued progression of vitiligo after completion of Nivolumab.

\section{Conflict of Interest Disclosures: None}

Funding: None

\section{Corresponding Author:}

Allison Good, MD

PGY-2 Dermatology Resident

University of Texas Medical Branch at Galveston

Department of Dermatology

301 University Blvd, 4.112 McCullough Bldg

Galveston, TX 77555-1327

Phone: (573) 808-0425

Fax: (409) 772-4456

E-mail: ajgood@utmb.edu

\section{References:}

1. Hua C, Boussemart L, Mateus C, Routier E, Boutros C, Cazenave $\mathrm{H}$, Viollet $\mathrm{R}$, Thomas M, Roy S, Benannoune N, Tomasic G, Soria JC, Champiat S, Texier M, Lanoy E, Robert C. Association of Vitiligo With Tumor Response in Patients With Metastatic Melanoma Treated With Pembrolizumab. JAMA Dermatol. 2016 Jan;152(1):45-51.

2. Koppolu V, Rekha Vasigala VK. Checkpoint immunotherapy by nivolumab for treatment of metastatic melanoma. J Cancer Res Ther. 2018 Oct-Dec;14(6):1167-1175.

3. Nakamura $Y$, Tanaka R, Asami $Y$, Teramoto $\mathrm{Y}$, Imamura $\mathrm{T}$, Sato $\mathrm{S}$, Maruyama $\mathrm{H}$, Fujisawa Y, Matsuya T, Fujimoto M, Yamamoto A. Correlation between vitiligo occurrence and clinical benefit in advanced melanoma patients treated with nivolumab: $\mathrm{A}$ multi-institutional retrospective study. $J$ Dermatol. 2017 Feb;44(2):117-122.

4. Yin ES, Totonchy MB, Leventhal JS. Nivolumab-associated vitiligo-like depigmentation in a patient with acute myeloid leukemia: A novel finding. JAAD Case Rep. 2017;3(2):90-92.

5. Lolli C, Medri M, Ricci M, et al. Vitiligo-like lesions in a patient treated with nivolumab for renal cell carcinoma. Medicine (Baltimore). 2018;97(52):e13810.

6. Frisoli ML, Essien K, Harris JE. Vitiligo: Mechanisms of Pathogenesis and Treatment. Annu Rev Immunol. 2020 Apr 26;38:621-648.

7. Kosche C, Mohindra N, Choi JN. Vitiligo in a patient undergoing nivolumab treatment for non-small cell lung cancer. JAAD Case Rep. 2018 Nov 10;4(10):1042-1044.

8. Edmondson LA, Smith LV, Mallik A. Nivolumab-induced vitiligo in a metastatic melanoma patient: A case report. $\mathrm{J}$ Oncol Pharm Pract. 2017 Dec;23(8):629-634. 